\title{
Introduction to the HICSS-50 Minitrack on Innovative Behavioral IS Security and Privacy Research
}

\author{
Merrill Warkentin \\ Mississippi State University \\ m.warkentin@msstate.edu
}

\author{
Anthony Vance \\ University of Hawai'i at Mānoa \\ anthony@,vance.name
}

\author{
Allen C. Johnston \\ University of Alabama \\ ajohnston@cba.ua.edu
}

This minitrack provides a venue for innovative research that rigorously addresses the risks to information system security and privacy, with a specific focus on individual behaviors within this nomological net. Domains include work related to detecting, mitigating, and preventing both internal and external human threats to organizational security. Papers may include theory development, empirical studies (both quantitative and qualitative), case studies, and other high-quality research manuscripts.

This year, the minitrack features nine papers addressing a range of behavioral security and privacy research questions that will stimulate further discussion and exploration of the key phenomena within this domain.

One group of papers addresses research issues related to privacy, including an in-depth literature review of the dependent variables, an exploration of privacy within the cloud environment, and an application of construal level to explore whether psychological distance is a factor.

- Wirth, Jakob. "Dependent Variables in the Privacy-Related Field: A Descriptive Literature Review"

- $\quad$ Lang, Michael, Manuel Wiesche, \& Helmut Krcmar. "Perceived Control and Privacy in a Professional Cloud Environment"

- Bandara, Ruwan, Mario Fernando, \& Shahriar Akter. "Is the Privacy Paradox a Matter of Psychological Distance? An Exploratory Study of the Privacy Paradox from a Construal Level Theory Perspective"

A second group of papers featured within this minitrack is focused on research topics related to human cognition issues in security and privacy, including the rational and non-rational theories of human decision making processes involved in security policy violations, the impact of variations in video design factors on perceptions of risk, benefits and information disclosure decisions, and the influence of online crowdedness on self-disclosure behavior.

- $\quad \mathrm{Xu}$, Zhengchaun \& Qing Hu. "The Role of Rational Calculus in Controlling Individual Propensity toward Information Security Policy Non-Compliance Behavior"

- $\quad$ Keith, Mark J., Jacob T. Frederickson, James Reeves, \& Jeffry Babb. "Optimizing Privacy Policy Videos to Mitigate the Privacy Policy Paradox"

- Choi, HanByeol, Chanhee Kwak, Junyeong Lee, \& Heeseok Lee. "Too Crowded to Disclose! Exploring the Relationship between Online Crowdedness and Self-Disclosure"

Finally, a third group also includes three papers. These involve engaging individuals in securityrelated issues, such as through misinformation campaigns, or more positively, through interaction with security professionals to address organizational and industry needs.

- $\quad$ Michele Maasberg, Emmanuel Ayaburi, Charles Liu, \& Yoris Au. "Exploring the Propagation of Fake Cyber News: An Experimental Approach"

- Pam Rowland, Ashley Podhradsky, \& Shephanie Plucker. "CybHER: A Method for Empowering, Motivating, Educating and Anchoring Girls to a Cybersecurity Career Path"

- $\quad$ Teodora Tatu, Clara Ament, \& Lennart Jaeger. "Lessons Learned from an Information Security Incident: A Practical Recommendation to Involve Employees in Information Security"

We trust that this year's contributions will stimulate interesting discussion and will advance the research within our discipline. 